\title{
Clusters of Multivariate Stationary Time Series by Differential Evolution and Autoregressive Distance
}

\author{
Roberto Baragona \\ Sapienza University of Rome, Dept. of Communication and Social Research \\ Via Salaria 113, Rome, Italy \\ roberto.baragona@uniroma1.it \\ http://w3.uniroma1.it/statstsmeh
}

\begin{abstract}
Clustering MTS is a difficult task that has to be performed in several application fields. We propose a method based on the coefficients of vector autoregressive (VAR) models and differential evolution (DE) that may be applied to sets of stationary MTS. Results from a simulation experiment that includes both linear and non linear MTS are displayed for comparison with genetic algorithms (GAs), principal component analysis (PCA) and the k-means algorithm. Part of the Australian Sign Language (Auslan) data are examined to show the comparative performance of our procedure on a real world data set.
\end{abstract}

Keywords: Autoregressive distance, Cluster analysis, Differential evolution, Multivariate time series.

\section{Introduction}

MTS are of chief interest in a large set of application fields, e.g. econometrics, environmental sciences, seismology, medicine, data mining, speech recognition, video image analysis. Surveys are for example [7] and [89]. Often data are collected by automatic devices (sensors, electronic transaction documents, the Internet) and a large amount of information is available. The standard organization for MTS is a rectangular array with $m$ rows (series components, variables, locations) and $n$ columns (observations taken at regular time intervals). Clustering is a common device which is used to reduce a large set of MTS to a smaller set of MTS representative of homogeneous groups. We assume that data are unlabeled, so that we have to check the existence and find the groups using only the available data. We examine in this paper the features that may be computed from the popular VAR model estimated matrices of coefficients. We observe for motivating such a choice that such features are simple and effective for clustering stationary MTS and may be easily and quickly computed by least squares. Many other features have been suggested. PCA of similarity matrices has been proposed for developing procedures for MTS classification 17,141]. Extensions of spectral measures for MTS have been discussed by [5]. The approach introduced by [9] splits the clustering procedure into two steps. In the first step each 
MTS is converted into a sequence of discrete values. In the second step either the dynamic time warping or a symmetric version of the Kullback-Leibler distance is computed from the discrete data. Many algorithms are available to cluster a set of features, in general a set of unlabeled data (see, e.g., [6]). Most efficient specially for complicated large data set have been found the meta heuristic methods (see, e.g., [16]) which solve the clustering problems put in terms of an optimization problems with respect to some cluster internal validity index (see, e.g., 311]). In particular the most used meta heuristic for clustering maybe are the GAs often in the presence of multiple objective functions (see, e.g., [2]). However DE ([15]) seems more suited to deal with real valued potential solutions, and we suggest using this method for clustering MTS. The paper is organized as follows. In Sect. 2 the features that we use for clustering are introduced. The DE method is illustrated in Sect. 3. In Sect. 4 a simulation study is reported that support the use of the AR weights and DE for clustering MTS. An application to a well-known set of real world data is presented in Sect. 5. Sect. 6] concludes.

\section{The Autoregressive Weights for Multivariate Time Series}

Let $x_{t}=\left(x_{1, t}, x_{2, t}, \ldots, x_{m, t}\right)^{\prime}$, where $t$ is a discrete valued time index, be a real valued $m$-dimensional MTS which admits the VAR infinite representation

$$
x_{t}=\sum_{i=1}^{\infty} \Pi_{i} x_{t-i}+a_{t},
$$

where the $\Pi_{i}$ are $m \times m$ matrices of coefficients and $a_{t}$ is a $m$-dimensional white noise with zero mean and variance covariance matrix $\Sigma_{a}$. Examples of such MTS are the popular finite order stationary VAR, the invertible vector movingaverage (VMA) and the finite order stationary and invertible vector $\mathrm{AR}$ and MA (VARMA) models. Given a MTS data set $\left\{x_{1}, x_{2}, \ldots, x_{n}\right\}$ a finite order $L$ VAR may be fitted to $x_{t}$ with $L$ large enough to obtain a good approximation of Eqn. (1). 10 suggested a test of hypothesis for every pair of MTS in a given set based on the VAR parameter coefficient matrices estimates $\hat{\Pi}_{i}, i=1, \ldots, L$. Let $\hat{\pi}_{i}$ be the column vector obtained by stacking the columns of $\hat{\Pi}_{i}, i=1, \ldots, L$. A set of measurements that may be used to characterize the MTS is the vector $\hat{\pi}=\left(\hat{\pi}_{1}^{\prime}, \hat{\pi}_{2}^{\prime}, \ldots, \hat{\pi}_{L}^{\prime}\right)^{\prime}$. These measurements may be viewed as an extension to the multivariate framework of the AR distance index introduced by $[12$ for univariate time series. We call these measurements as the features of interest that may capture the characteristic behavior of each MTS in a given set. A distance measure, e.g. the Euclidean metric, between two feature vectors may be assumed as the distance between the respective MTS.

\section{Differential Evolution Based Clustering}

Most clustering algorithms which include centers evolution use GAs. The contribution of the present paper aims at showing that DE is a better choice than GAs 
if, as it is the case with the AR distance here, chromosomes with real valued genes are employed. DE is a population based method that evolves a population of potential solutions through a sequence of generations. Each solution is a vector $x=\left(x_{1}, x_{2}, \ldots, x_{m}\right)^{\prime} \in R^{m}$ which at each generation undergoes the DE process. A vector $v_{0}$ (called the base vector) different from $x$ is selected randomly in the population. Then two other vectors $v_{1}$ and $v_{2}$ are randomly selected and their difference is scaled by the scale factor $F \in(0,1)$. A new potential solution $u$ called mutant is computed $u=v_{0}+F\left(v_{1}-v_{2}\right)$ and recombined with the initial individual vector $x$ to produce an offspring vector $y$ by means of uniform crossover. This means that each element of $y$ is assumed equal to the corresponding element of the mutant $u$ with a fixed probability $p_{c}$ or equal to the original element of the vector $x$ with probability $\left(1-p_{c}\right)$. Finally $y$ replaces $x$ in the population if a better value of the objective function is obtained. Basically the population size remains the same during the whole DE process. More details and variants may be found in 13 . For implementing the examples in the following Sect. 4 and 5 we used the Matlab code from [13] modified for the purpose of clustering MTS. The population consists in a set of vectors with length $g \times m^{2} L$, where $g$ is the number of cluster and $m^{2} L$ the total number of the VAR coefficients. Each vector encodes a sequence of $g$ centers that represent clusters of the sequences of AR weights computed from the MTS data set. We implemented the fuzzy clustering method with objective function the FCM index $J_{m}$ for which GAs have been proposed ([3]). The centers evolution includes the update that takes place in the FCM algorithm as soon as the new clusters have been defined and produces an improvement of the rate of convergence to the optimal solution. The same device has been included in the GAs clustering that we use for performance comparison.

\section{Experiments on Artificial Data Sets}

In the following simulation experiments the AR weights are computed by fitting a $\operatorname{VAR}(L)$ to each artificial MTS in a given set. Then we compare the procedure based on DE, GAs, PCA and the k-means algorithm. Comparison is made in terms of the misclassification error rate (MSCR) and the modified Rand index (MRI) (4]). As a first experiment, a set of 2-dimensional artificial time series data of length 100 has been generated as in [9] from the bivariate VARMA models

$$
x_{t}-\mu=\Phi\left(x_{t-1}-\mu\right)+a_{t}-\Theta a_{t-1},
$$

where $\mu=(10,20)^{\prime}$,

$$
\Phi=\left(\begin{array}{cc}
1.2 & -0.5 \\
0.6 & 0.3
\end{array}\right), \quad \Theta=\left(\begin{array}{cc}
-0.6 & 0.3 \\
0.3 & 0.6
\end{array}\right),
$$

and $\left\{a_{t}\right\}$ is a sequence of independent identically normally distributed vector random variables with mean zero and variance covariance matrix

$$
\Sigma=\left(\begin{array}{cc}
1.0 & 0.5 \\
0.5 & 1.25
\end{array}\right) \text {. }
$$


Three clusters were produced, the first one by the $\operatorname{VAR}(1)$ obtained by setting in Eqn. (2) $\Theta=0$, the second one by the $\operatorname{VMA}(1)$ with $\Phi=0$, and the third one by the full VARMA(1,1) model (2). The procedure suggested in Liao (2007) is reported to produce, in 9 runs, some misclassification that arises from confusion between the $\operatorname{VAR}(1)$ model and the $\operatorname{VARMA}(1,1)$ model. We generated 300 artificial MTS, 100 for each cluster. We run our procedure for 1000 replications using the features computed as the AR weights obtained by fitting a $\operatorname{VAR}(2)$ model to each MTS. On such features the DE, GA, PCA and k-means clustering algorithms have been applied. The three clusters have been recovered almost exactly by the first two algorithms. PCA and k-means produce inferior results, with MSCR about $1 \%$ and MRI slightly less than 0.9 . We run a similar but more difficult simulation experiment by generating 3-dimensional time series and using in Eqn. (2) a stronger AR structure and a weaker MA structure, so as to make more likely a possible confusion between MTS in clusters 1 and 3 . We obtained the results displayed in Table 1. According to both cluster external validity indexes the DE gives better results than GA in terms of both average and standard error. The 3 clusters are recovered satisfactorily with MSCR about $3 \%$, and MRI greater than 0.9 . Both PCA and k-means give inferior results in all respects. Then the proposed procedure has been checked on a set of non linear MTS generated by 2 two-regime bivariate threshold models (VTAR). The VAR features have been computed of order 5 and DE performance is quite satisfactory as the cluster structure is exactly recovered while other algorithms show higher MSCR and lower MRI. This experiment shows that our method is able to deal with non linear as well with linear MTS. For all simulation experiments we may explain the better performance of DE based clustering of MTS VAR coefficients as in DE the feasible solutions are put in terms of real valued alleles. DE has been developed since its beginning to deal with real valued solutions while theory and practice of GAs mainly rely on binary encoding of the solutions. On the other hand, clustering MTS by using PCA reduces the size of the data set and saves computation time but it seems unable to distinguish between underlying models with an accuracy comparable to GAs or DE.

Table 1. Clustering results for 3-dimensional MTS data set

\begin{tabular}{lll}
\hline Algorithm & MSCR & MRI \\
\hline DE & 0.0272 & 0.9227 \\
& $(0.0181)$ & $(0.0383)$ \\
GA & 0.0332 & 0.9169 \\
& $(0.0527)$ & $(0.0654)$ \\
PCA & 0.1005 & 0.8698 \\
& $(0.1848)$ & $(0.2082)$ \\
k-means & 0.1563 & 0.7880 \\
& $(0.2063)$ & $(0.2201)$ \\
\hline
\end{tabular}

The standard errors of the estimates are reported in parentheses. 


\section{The Australian Sign Language Data}

This data consists of sample of Auslan signs. 27 examples of each of 95 Auslan signs were captured from a native signer using high-quality position trackers. Each vector time series has 15 components series while the length varies from 45 to 60 data points. The data have been downloaded from the UCI Machine Learning Repository (http://archive.ics.uci.edu/ml). As in 9] only 5 samples of each of the words 'alive', 'all', 'boy', 'cold' from the directory 'john4' have been used for illustration purpose and only the first 11 numeric variables are used. However we considered only the variables 1-4 to avoid singularity of the variance covariance matrices, as variables 5 and 6 have constant values equal to -1 and variables $7-11$ have values that are very similar each other. The DE, GA, PCA and k-means algorithms have been applied on the feature set which included the VAR weights computed as the estimates of the matrix coefficients of a $\operatorname{VAR}(2)$ model fitted to each one of the 204 -dimensional MTS. On Table 2 results are evaluated according to the MSCR and the MRI. DE outperforms the remaining algorithms. The word 'cold' is correctly recovered by both DE and GA for all the 5 samples. The words 'alive' and 'all' are recognized for 4 out of 5 samples and the word 'boy' for 3 out of 5 samples by DE. The GA, on the other hand, displays some confusion between the words 'alive' and 'boy'. The procedure suggested by 9] too makes a specific error in mistaking one of the 'boy' signs as an 'all' sign.

Table 2. Clustering algorithms applied to 20 samples from the Auslan data set

\begin{tabular}{lll}
\hline Algorithm & MSCR & MRI \\
\hline DE & 0.2000 & 0.5136 \\
GA & 0.4500 & 0.2344 \\
PCA & 0.5000 & 0.2073 \\
k-means & 0.5500 & 0.1979 \\
\hline
\end{tabular}

\section{Conclusions}

A method based on AR distance and DE has been proposed for clustering stationary MTS. Results from a simulation experiment seem quite satisfactory in comparison with GAs and PCA based clustering and the k-means algorithm and support the use of our procedure for clustering linear and non linear MTS. The execution time for a set of 300 MTS with 100 observations each is on the average $0.359 \mathrm{sec}$ for feature extraction while, for the clustering procedure, $1.578 \mathrm{sec}$ for DE, $3.094 \mathrm{sec}$ for GA, $0.871 \mathrm{sec}$ for PCA and $0.015 \mathrm{sec}$ for k-means. So DE seems to attain a fair trade-off between speed and performance. An application to some data from the Auslan data set confirms the better performance of our procedure with respect to competing alternatives. 
Acknowledgments. Research financially supported by 'Ministero dell'Istruzione, Universitá e Ricerca Scientifica', Italy, through grant 'Progetto di Interesse Nazionale 2007' PRIN2007.

\section{References}

1. Allefeld, C., Bialonski, S.: Detecting syncronization clusters in multivariate time series via coarse-graining of Markov chains. Tech. rep., arXiv:0707.2479v1 [physics.data-an] (July 17, 2007)

2. Bandyopadhyay, S., Baragona, R., Maulik, U.: Clustering multivariate time series by genetic multiobjective optimization. Metron 68, 161-183 (2010)

3. Bezdek, J.C., Pal, N.R.: Some new indexes of cluster validity. IEEE T. Sist. Man Cyb. - Part B: Cybernetics 28, 301-315 (1998)

4. Hubert, L., Arabie, P.: Comparing partitions. J. Classif. 2, 193-218 (1985)

5. Kakizawa, Y., Shumway, R.H., Taniguchi, M.: Discrimination and clustering for multivariate time series. J. Am. Stat. Assoc. 93, 328-340 (1998)

6. Kaufman, L., Rousseeuw, P.J.: Finding Groups in Data. Wiley, New York (2005)

7. Keogh, E., Kasetty, S.: On the need for time series data mining benchmarks: A survey and empirical demonstration. Data Min. Knowl. Disc. 7, 349-371 (2003)

8. Liao, T.W.: Clustering of time series data - a survey. Pattern Recogn. 38, 1857-1874 (2005)

9. Liao, T.W.: A clustering procedure for exploratory mining of vector time series. Pattern Recogn. 40, 2550-2562 (2007)

10. Maharaj, E.A.: Comparison and classification of stationary multivariate time series. Pattern Recogn. 32, 1129-1138 (1999)

11. Pakhira, M.K., Bandyopadhyay, S., Maulik, U.: Validity index for crisp and fuzzy clusters. Pattern Recogn. 37, 487-501 (2004)

12. Piccolo, D.: A distance measure for classifying ARIMA models. J. Time Ser. Anal. 11, 153-164 (1990)

13. Price, K.V., Storn, R., Lampinen, J.: Differential evolution, a practical approach to global optimization. Springer, Berlin (2005)

14. Singhal, A., Seborg, D.E.: Clustering multivariate time-series data. J. Chemometr. 19, 427-438 (2005)

15. Storn, R., Price, K.: Differential evolution - a simple and efficient heuristic for global optimization over continuous spaces. J. Global Optim. 11, 341-359 (1997)

16. Winker, P.: Optimization heuristics in econometrics: applications of threshold accepting. Wiley, Chichester (2001)

17. Yang, K., Shahabi, C.: A PCA-based similarity measure for multivariate time series. In: MMDB 2004: Proceedings of the 2nd ACM international workshop on Multimedia databases, pp. 65-74. ACM, New York (2004) 